\begin{tabular}{|c|c|c|c|}
\hline & $\begin{array}{c}\text { ITT/BRCAwt* } \\
n=473\end{array}$ & $\begin{array}{c}\mathrm{HRd} / B R C A w t \\
n=150\end{array}$ & $\begin{array}{c}\text { HRp/BRCAwt } \\
n=249\end{array}$ \\
\hline Hazard ratio $(95 \% \mathrm{Cl})$, & $0.69(0.54-0.88)$ & $0.50(0.31-0.83)$ & $0.68(0.49-0.94)$ \\
\hline \multicolumn{4}{|l|}{ mPFS, months } \\
\hline Niraparib & 10.9 & 19.6 & 8.1 \\
\hline Placebo & 7.4 & 8.2 & 5.4 \\
\hline$\triangle \mathrm{mPFS}$, months & 3.5 & 11.4 & 2.7 \\
\hline \multicolumn{4}{|c|}{$\begin{array}{l}\text { *Includes patients who were HRnd but known to be BRCAwt. } \\
\text { HRd, homologous recombination deficient; HRnd, homologous recombination not determined; } \\
\text { HRp, homologous recombination proficient; ITT, intention-to-treat; mPFS, median } \\
\text { progression-free survival; wt, wild-type. }\end{array}$} \\
\hline
\end{tabular}

Conclusion Niraparib improved PFS when utilised as maintenance therapy after front-line treatment of OC in patients with BRCAwt tumours, including in the most difficult to treat subgroup of patients with BRCAwt and HRp tumours.

Disclosures Dr. Braicu reports honoraria from AstraZeneca, Tesaro, GSK, Roche Pharma, Clovis and MSD; consulting or advisory roles at AstraZeneca, Tesaro, GSK, Roche Pharma, Clovis, MSD, Abbvie, Eisai, Immunogen, Takeda; institutional research funding from Roche Diagnostics and Takkeda; and travel, accommodation and expenses from Astra Zeneca, RochePharma, Clovis, and MSD.

Dr. Pothuri reports grants, personal fees and non-financial support from GSK; Advisory Board fees from AstraZeneca and Clovis Oncology.

Dr. Alejandro Perez-Fidalgo reports speaker fees from GSK, AstraZeneca, Clovis, and Roche; advisory fees from GSK, AstraZeneca, Clovis, Abilify Pharma, Clinigen, Roche, and Amgen; grant from GSK; and travel fees from AstraZeneca and Roche.

Dr. O'Malley reports personal fees from Immunogen, Eisai, Agenus, GSK : Consultant/Advisory Board for Clovis, Ambry, Abbvie, Janssen/J\&J, Regeneron, Novacure, Myraid Genetics, Tarveda, Amgen, VentiRx, Array Biopharma, EMD Serono, Ergomed; Steering committee for Genentech/Roche and Merck; Institutional funding from Ajinomoto Inc, Ludwig Cancer Research, Stemcentrx, Inc, CERULEAN PHARMA, GOG Foundation, BMS, Serono Inc, TRACON Pharmaceuticals, Yale University, New Mexico Cancer Care Alliance, INC Research, Inc., Inventiv Health Clinical, Iovance Biotherapeutics, Inc, and PRA International.

Dr. Graybill reports personal fees from GSK.

Dr. Dahlstrand reports personal fees from AstraZeneca and Roche.

Dr. Monk reports consulting and advisory role at Merck, GSK, Roche/Genentech, AstraZeneca, Advaxiz, Cerulean Pharma, Amgen, Immunogen, NuCana BioMed, Clovis Oncology, Pfizer, Mateon Therapeutics, Precision Oncology, Perthera, Abbvie, Myriad Pharmaceuticals, Incyte, VBL Therapeutics, Takeda, Samumed, Oncomed, OncoSec, ChemoID, Geistlich Pharma, Eisai and Chemocare; Speakers' bureau at Roche/Genentech, AstraZeneca, Janssen, Clovis Oncology and GSK; Honoraria from Merck, GSK, Roche/Genentech, AstraZeneca, Advaxis, Immunogen, NuCana BioMed, Clovis Oncology, Pfizer, Mateon Therapeutics, Precision Oncology, Pethera, Abbvie, Myriad Pharmaceuticals, Incyte, Janssen, Amgen, Genmab, Samumed, Takeda, VBL Therapeutics,
Puma Biotechnology, Immunomedics, Conjupro Biotherapeutics, Agenus, OncoQuest, ChemoID, Geistlich Pharma, Eisai and Chemocare; and Research funding from Novartis, Amgen, Genentech, Lilly, Janssen, Array BioPharma, GSK, Morphotek, Pfizer, Advaxis, AstraZeneca, Immunogen, Regeneron, and Nucana.

Drs. Honhon and Fabbro have nothing to disclose.

Dr. Gupta is an employee of GlaxoSmithKline.

\section{FEASIBILITY STUDY OF A NETWORK META-ANALYSIS AND UNANCHORED POPULATION-ADJUSTED INDIRECT TREATMENT COMPARISON OF NIRAPARIB, OLAPARIB, AND BEVACIZUMAB AS MAINTENANCE THERAPIES IN PATIENTS WITH NEWLY DIAGNOSED ADVANCED OVARIAN CANCER}

${ }^{1}$ Domenica Lorusso, ${ }^{2}$ Holly Guy, ${ }^{3}$ Jean Hamilton, ${ }^{4}$ Yevgeniy Samyshkin, ${ }^{5}$ Karin Travers, ${ }^{4}$ Carol Hawkes, ${ }^{6}$ Robert L Coleman. 'Gynaecology Oncology Unit, Fondazione IRCCS National Cancer Institute of Milan, Milan, Italy; ${ }^{2}$ Fiecon, London, UK; ${ }^{3}$ School of Health and Related Research, University of Sheffield, Sheffield, UK; ${ }^{4}$ GlaxoSmithKline, London, UK; ${ }^{5}$ GlaxoSmithKline, Waltham, MA, USA; 6 University of Texas MD Anderson Cancer Center, Houston, TX

\subsection{6/ijgc-2020-ESG0.125}

Introduction/Background Although randomised controlled trials (RCTs) have demonstrated the benefit of PARP inhibitors and bevacizumab as monotherapies and combination therapies, there is limited direct head-to-head evidence of their relative clinical efficacy.

In the PRIMA study, niraparib demonstrated a clinically significant improvement in progression-free survival (PFS) compared with placebo, as a first-line (1L) ovarian cancer (OC) maintenance therapy.

The objectives of the study were to assess feasibility of an indirect treatment comparison (ITC) and a population-adjusted indirect treatment comparison (PAIC) for estimating the relative efficacy of niraparib compared with olaparib, olaparib plus bevacizumab, and bevacizumab as maintenance following $1 \mathrm{~L}$ chemotherapy in OC. The study focused on fully powered statistical cohorts.

Methodology Trials included in the ITC analysis were based on a systematic literature review conducted in February 2020.

Guidelines from the Cochrane Handbook for Systematic Reviews of Interventions were used to assess the level of heterogeneity across the studies in terms of designs, population characteristics, treatment arms and outcome measures. 
The feasibility of PAIC for the PRIMA and PAOLA-1 trials was assessed based on assumptions outlined in the guidance by the Decision Support Unit in NICE DSU Technical Support Document 18; PFS was the outcome for the analysis.

Results All 12 RCTs assessed for ITC feasibility were excluded based on various factors including: the lack of a common comparator with PRIMA within the network (ICON-7, GOG0218, PAOLA-1, VELIA/GOG-3005); differing measurement of PFS and overall survival starting timepoint due to trial design (ICON-7, GOG-0218, VELIA/GOG-3005); inclusion of stage III patients with no visible residual disease following debulking surgery (PAOLA-1, SOLO-1, VELIA/GOG-3005); disparity between disease biomarker (SOLO-1).

For the PAIC, three fundamental differences between the PRIMA and PAOLA trials were identified; inclusion criterion related to residual disease was wider in PAOLA meaning that the 'conditional constancy of absolute effects' was violated; receipt of neoadjuvant chemotherapy was identified as a confounding factor that would bias a PAIC; discrepancies in the assessment of type and frequency of measurement of the PFS outcome.

Conclusions Based on the evidence currently available, neither an ITC nor PAIC would meet current guidelines, such as those outlined by the International Society for Pharmacoeconomics and Outcomes Research, for these analyses. Their results would not be considered appropriate evidence for use in clinical decision making or reimbursement decisions. The extent of imbalance caused by differences in the patient inclusion/exclusion criteria for intended comparisons is unknown and a recognised limitation of PAICs.

Disclosures This study was funded by GlaxoSmithKline.

Due to lengthy author disclosures, author COI information will be provided directly to the congress.

\section{THE USE OF REAL-WORLD EVIDENCE FROM THE EDINBURGH OVARIAN CANCER DATABASE TO EXPLORE A DATA GAP IN THE PRIMA TRIAL}

${ }^{1}$ Robert L Hollis, ${ }^{2}$ Zsofia Kiss, ${ }^{2}$ Nichola Roebuck, ${ }^{2}$ Helen Starkie-Camejo, ${ }^{2}$ Kiera Heffernan, ${ }^{1}$ Charlie Gourley. ${ }^{1}$ Nicola Murray Centre for Ovarian Cancer Research, Cancer Research UK Edinburgh Centre, MRC Institute of Genetics and Molecular Medicine, Edinburgh, UK; ${ }^{2}$ GlaxoSmithKline Brentford, Middlesex, London, UK

10.1136/ijgc-2020-ESG0.126
Introduction/Background Niraparib is an oral, highly selective poly(ADP-ribose) polymerase inhibitor (PARPi). The PRIMA/ ENGOT-OV26/GOG-3012 trial intention-to-treat population included stage III and IV ovarian cancer (OC) patients postprimary or interval debulking surgery (PDS/IDS) irrespective of residual disease (visible residual disease [VRD] or no VRD [NVRD]), except for stage III patients post-PDS with NVRD. A data gap therefore exists in the stage III postPDS NVRD population. This study aims to use real-world evidence from the Edinburgh Ovarian Cancer Database to explore the difference in overall survival (OS) and progression-free survival (PFS) in: a) a 'simulated-PRIMA cohort' (stage III VRD after PDS, stage III and IDS, and stage IV OC), b) a 'simulated-NVRD after PDS cohort' (stage III NVRD after PDS) and c) a larger 'simulated-broader cohort' (both cohorts $\mathrm{a}$ and $\mathrm{b}$ plus the additional stage III nonevaluable debulking status cases).

Methodology A retrospective observational study was conducted to examine characteristics and long-term outcomes for patients diagnosed with advanced OC within the Edinburgh Cancer Centre between 1 January 2000 and 31 December 2015. Patients were followed until their last patient record or last data cut (January 2019) and were chosen to match the three defined populations. Main outcomes were OS and PFS, the latter defined as time from diagnosis to first progression as defined by radiology, tumour marker (CA125) or the treating physician where other investigations were not evaluable.

Results Baseline patient characteristics in the simulated-PRIMA $(\mathrm{n}=472)$, simulated-stage III NVRD after PDS $(\mathrm{n}=69)$ and simulated-broader $(n=569)$ cohorts matched the PRIMA trial in most categories. PFS and OS for simulated cohorts are shown in the table 1 . When compared with the simulatedPRIMA cohort, the simulated-stage III NVRD after PDS

Abstract 367 Table 1
\begin{tabular}{|l|c|c|}
\hline Cohort & Median PFS, yr & Median os, $\mathbf{y r}$ \\
\hline Simulated-PRIMA ( $n=472)$ & 1.20 & 2.71 \\
\hline Simulated-stage III NVRD after PDS ( $n=69)$ & 2.45 & 6.84 \\
\hline Simulated-broader ( $n=569$ ) & 1.26 & 3.07 \\
\hline NVRD, no visible residual disease; OS, overall survival; PDS, primary debulking surgery; \\
PFS, progression-free survival.
\end{tabular}

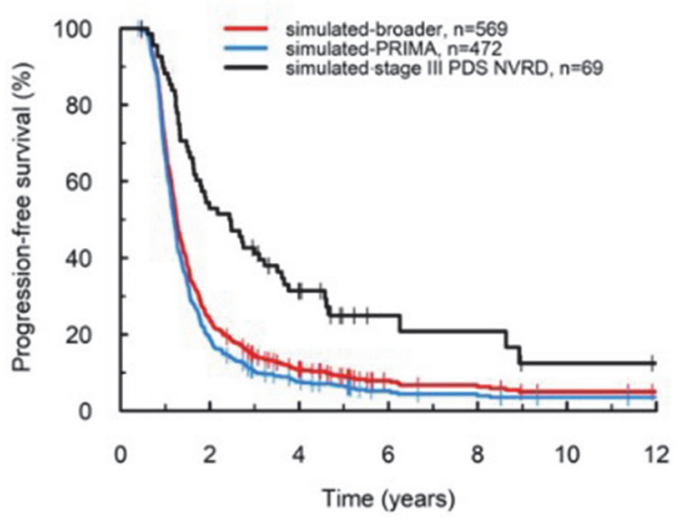

